\title{
Neutron Diffraction Study of Load Response and Residual Stresses in WC- (Ni/Co) Composites
}

\author{
Jon W. Paggett \\ Dr. Aaron D. Krawitz, Dissertation Supervisor
}

\begin{abstract}
The in-situ response of WC-Ni and WC-Co composites under compressive loading was measured using neutron diffraction techniques. These measurements give a clear indication of the elastic strain behavior of both the carbide and metal binder phases in directions both axial and transverse to the applied load. Residual stress measurements were also made on as-produced samples and on those samples which had been mechanically loaded. These measurements show a substantial relaxation in the initial thermal residual stresses of the cemented carbides with high binder content, most of which occurs by the end of the first load-unload cycle. This relaxation in residual stress is also found to be anisotropic, relaxing more in the direction transverse to the loading axis than in the direction parallel to the loading axis. Strain distribution was considered by observing the change in peak widths in the diffraction data. Taken as a whole, the data suggests a profound role for thermal residual stress and the point-to-point variation of residual stress in the response of cemented carbides to applied load. Localized yielding on the scale of the microstructure, which acts to absorb energy, is proposed as the mechanism that accounts for the superior toughness of these materials.
\end{abstract}

